\section{Structure of the Spt16 Middle Domain Reveals Functional Features of the Histone Chaperone FACT*}

Received for publication, January 8, 2013, and in revised form, February 5, 2013 Published, JBC Papers in Press, February 15, 2013, DOI 10.1074/jbc.C113.451369

David J. Kemble ${ }^{\ddagger}$, Frank G. Whitby ${ }^{\ddagger}$, Howard Robinson ${ }^{\S}$, Laura L. McCullough ${ }^{\ddagger}$, Tim Formosa ${ }^{\ddagger 1}$, and Christopher P. Hill ${ }^{\ddagger 2}$

From the ${ }^{\ddagger}$ Department of Biochemistry, University of Utah School of Medicine, Salt Lake City, Utah 84112-5650 and the ${ }^{\S}$ Biology Department, Brookhaven National Laboratory, Upton, New York 11973-5000

Background: Spt16-M is a functionally important region of the essential histone chaperone FACT.

Results: The Spt16-M crystal structure was determined, and mutations that affect FACT function were mapped to this double $\mathrm{PH}$ domain.

Conclusion: Spt16-M resembles the Pob3 and Rtt106 histone chaperones and binds histones $\mathrm{H} 3-\mathrm{H} 4$.

Significance: Mechanistic models of FACT are advanced, published genetic data are explained, and functionally important surfaces are identified.

The histone chaperone FACT is an essential and abundant heterodimer found in all eukaryotes. Here we report a crystal structure of the middle domain of the large subunit of FACT (Spt16-M) to reveal a double pleckstrin homology architecture. This structure was found previously in the Pob3-M domain of the small subunit of FACT and in the related histone chaperone Rtt106, although Spt16-M is distinguished from these structures by the presence of an extended $\alpha$-helix and a C-terminal addition. Consistent with our finding that the double pleckstrin homology structure is common to these three histone chaperones and reports that Pob3 and Rtt106 double pleckstrin homology domains bind histones $\mathrm{H} 3-\mathrm{H} 4$, we also find that Spt16-M binds $\mathrm{H} 3-\mathrm{H} 4$ with low micromolar affinity. Our structure provides a framework for interpreting a large body of genetic data regarding the physiological functions of FACT, including the identification of potential interaction surfaces for binding histones or other proteins.

FACT (faciltiates chromatin transactions) is an abundant, highly conserved, and essential heterodimeric histone chaperone composed of Spt16 and either Pob3 (yeast) or the related SSRP1 (higher eukaryotes) (1-3). FACT has been implicated in

* This work was supported, in whole or in part, by National Institutes of Health Grants R01 GM59135 (to C. P. H. and T. F.) and GM064649 (to T. F.).

The atomic coordinates and structure factors (code 4IOY) have been deposited in the Protein Data Bank (http://wwpdb.org/).

${ }^{1}$ To whom correspondence may be addressed. Fax: 801-581-7959; E-mail: tim@biochem.utah.edu.

${ }^{2}$ To whom correspondence may be addressed. Fax: 801-581-7959; E-mail: chris@biochem.utah.edu. many chromatin-related processes, including promoter recognition, transcription initiation site selection, transcription elongation, maintenance of repressive chromatin, replacement of nucleosomes following transcription, incorporation of histone variants into chromatin, initiation of DNA replication, and progression of DNA replication complexes (1-3). Purified FACT induces or stabilizes an alternative form of nucleosomes in vitro in which DNA accessibility is dramatically enhanced without loss of histone protein components (4). This reorganizing activity is thought to be central to its participation in a broad range of in vivo processes that depend on nucleosome dynamics, with the reversibility of the reaction allowing FACT to promote either nucleosome assembly or disassembly in different circumstances.

Human FACT binds to $\mathrm{H} 2 \mathrm{~A}-\mathrm{H} 2 \mathrm{~B}$ and $\mathrm{H} 3-\mathrm{H} 4$ in vitro, with higher affinity for $\mathrm{H} 2 \mathrm{~A}-\mathrm{H} 2 \mathrm{~B}(5,6)$. Current models suggest that FACT maintains contact with multiple components of nucleosomes simultaneously, preventing dispersal of the nucleosome during transcription $(1,7)$. Consistent with this view, FACT is composed of multiple structural domains that can be detached from one another by limited proteolysis and are well behaved when expressed independently $(8,9)$. The structures of several of these domains have been determined, including the N-terminal domain of Spt16 (Spt16-N) and the N-terminal/ dimerization and middle domains of Pob3 (Pob3-N/D and Pob3-M) (9-12) (see Fig. 1A). Spt16-N has a pita-fold structure that suggests a peptide binding function, but the physiological ligand and role of this nonessential domain remain uncertain. The Pob3-N/D domain forms a pleckstrin homology $(\mathrm{PH})^{3}$ domain (Protein Data Bank (PDB) code 3F5R), and the Pob3-M domain comprises two $\mathrm{PH}$ domains that are fixed rigidly to one another in an unusual arrangement called the double pleckstrin homology domain (9). Notably, the related H3-H4 chaperone Rtt106 also forms a double PH domain, and models for the Rtt106 interaction with $\mathrm{H} 3-\mathrm{H} 4$ have been proposed $(13,14) . \mathrm{PH}$ and double $\mathrm{PH}$ domains, which often share little homology with one another at the primary sequence level and are therefore difficult to recognize in the absence of structural information, are therefore emerging as common elements of histone chaperones.

Genetic studies have indicated that the Spt16-M domain has key roles in the physiological function of FACT because many of the mutations that affect FACT function map to this domain $(15,16)$. Mutations in Spt16-M can be suppressed by altering histones to destabilize the interface between $\mathrm{H} 2 \mathrm{~A}-\mathrm{H} 2 \mathrm{~B}$ dimers and $\mathrm{H} 3-\mathrm{H} 4$, and mutations in $\mathrm{H} 3$ can be suppressed by mutations in Spt16-M $(15,17,18)$. Spt16-M therefore appears to make important contacts with histones during nucleosome reorganization by FACT, but more detailed mechanistic insights have been unavailable due to the lack of structural information. Here, we report a crystal structure of Spt16-M

\footnotetext{
${ }^{3}$ The abbreviations used are: $\mathrm{PH}$, pleckstrin homology; TEV, tobacco etch virus; r.m.s.d., root mean square deviation; $S A D$, single anomalous diffraction; FA, fluorescence anisotropy.
} 


\begin{tabular}{|c|c|c|}
\hline & Spt16, native & Spt16, SeMet \\
\hline Data collection & Home source & NSLS $\times 29$ \\
\hline Space group & $\mathrm{P}_{5}$ & $\mathrm{P}_{5}$ \\
\hline Cell dimensions: $a, b, c(\AA)$ & $134.2,134.2,40.3$ & $133.5,133.5,40.5$ \\
\hline Resolution $(\AA)$ & $30.0-1.95(2.02-1.95)$ & $40.0-2.60(2.69-2.60)$ \\
\hline No. of reflections total unique & $256,360(29,648)$ & $260,874(24,884)$ \\
\hline Wavelength $(\AA)$ & 1.5418 & 0.9792 \\
\hline $\mathrm{I} / \sigma_{\mathrm{I}}$ & $8(1.5)$ & $10(2.1)$ \\
\hline Mosaicity $\left({ }^{\circ}\right)$ & 0.75 & 1.2 \\
\hline Completeness (\%) & $96.5(84.5)$ & $100(100.0)$ \\
\hline$R_{\mathrm{sym}}{ }^{a}(\%)$ & $0.078(0.517)$ & $0.143(0.912)$ \\
\hline \multicolumn{3}{|l|}{ Refinement } \\
\hline Resolution (high) & $30.0-1.95(2.02-1.95)$ & \\
\hline$R_{\text {work }} / R_{\text {free }} b, c(\%)$ & $0.177 / 0.197(0.262 / 0.320)$ & \\
\hline No. of protein atoms & 2,183 & \\
\hline No. of solvent atoms & 191 & \\
\hline No. of phosphates & 1 & \\
\hline r.m.s.d. bond lengths $(\AA) /$ angles $\left(^{\circ}\right)$ & $0.009 / 1.173$ & \\
\hline$\varphi / \psi$ most favored/allowed (\%) & $91.2 / 7.5$ & \\
\hline$\langle\mathrm{B}\rangle\left(\AA^{2}\right):$ protein/solvent & $59 / 54$ & \\
\hline
\end{tabular}

${ }^{a} R_{\text {sym }}=\left(\left|\left(\sum \mathrm{I}-\langle\mathrm{I}\rangle\right)\right|\right) /\left(\sum \mathrm{I}\right)$, where $\langle\mathrm{I}\rangle$ is the average intensity of multiple observations.

${ }^{b} R_{\text {work }}=\Sigma|| F_{o}|-| F_{c}|/ \Sigma| F_{o} \mid$, where $\left|F_{o}\right|$ and $\left|F_{c}\right|$ are the observed and calculated structure factor amplitudes, respectively.

${ }^{c} R_{\text {free }}=R_{\text {work }}$ calculated using a random set of reflections (5\% of total) that were not used in refinement calculations.

from Saccharomyces cerevisiae to $1.95 \AA$ resolution. Surprisingly, this domain adopts a double pleckstrin homology architecture similar to the Pob3-M and Rtt106 PH domains. We map the published genetic data to the structure, revealing features of Spt16-M that are important for the physiological functions of FACT. We also show that Spt16-M binds to H3-H4, although this binding does not fully recapitulate the stronger interaction between FACT and H3-H4. Our results therefore support the model that Spt16-M contributes to the FACT-histone interaction and that FACT uses multiple independent contacts to bind the components of nucleosomes.

\section{EXPERIMENTAL PROCEDURES}

Protein Expression and Purification-S. cerevisiae Spt16-M was inserted into the pET-151DTOPO vector and verified by DNA sequencing. This vector encodes an N-terminal $6 \times$ histidine tag followed by a tobacco etch virus (TEV) protease cleavage site, such that processing by TEV protease leaves Spt16 residues Gly-675-Asp-958 preceded by 8 non-native residues (GIDPFTHM) and missing the predicted flexible loop residues Gly-775-Ser-780, inclusive. Spt16 was expressed in Escherichia coli BL21-RIL CodonPlus cells (Stratagene) using the autoinduction method (19). Cells were lysed by sonication, and protein was purified by nickel chelation nitrilotriacetic acid chromatography. Protein was incubated overnight at $4{ }^{\circ} \mathrm{C}$ with histidine-tagged TEV protease followed by reincubation with nitrilotriacetic acid resin to remove the His-tagged TEV protease and uncleaved Spt16-M. Spt16 was further purified by anion exchange (HiTrap Q HP) and size exclusion chromatography (Superdex SD200).

Crystallization and Structure Determination-Spt16-M was crystallized by vapor diffusion in sitting drops against a well solution containing $0.1 \mathrm{M}$ sodium potassium phosphate, $\mathrm{pH}$ 6.0, $0.2 \mathrm{M} \mathrm{NaCl}, 40 \%$ PEG-400, and $10 \mathrm{~mm}$ tris(2-carboxyethyl)phosphine-HCl. Crystals were cryocooled by plunging into liquid nitrogen prior to data collection. Native data were collected with a copper rotating anode $\mathrm{x}$-ray source, and SeMet SAD data were collected at the National Synchrotron Light Source (NSLS), beamline X29. Data were processed using HKL2000 (Denzo and Scalepack) (20). The structure was determined by the SAD method and refined using PHENIX (21). COOT was used for model building (22). Figures of molecular structures were made using PyMOL (23). See Table 1 for crystallographic statistics.

Fluorescence Anisotropy-Oregon Green-labeled Xenopus laevis $\mathrm{H} 3-\mathrm{H} 4$ histones were prepared as described previously (4). H3-H4 (1 nM) was incubated for $1 \mathrm{~h}$ at $25^{\circ} \mathrm{C}$ with varying amounts of Spt16-M (0.24-504 $\mu \mathrm{M})$ in $25 \mathrm{~mm}$ Tris. $\mathrm{HCl}, \mathrm{pH} 7.5$, $150 \mathrm{~mm} \mathrm{NaCl}$, 5\% glycerol, $1 \mathrm{~mm} \mathrm{MgCl}_{2}$, and $1 \mathrm{~mm} 2$-mercaptoethanol. Data were measured on a Tecan Infinite $200 \mathrm{spec}-$ trometer with excitation/emission wavelengths of $485 \mathrm{~nm} / 535$ $\mathrm{nm}$. Raw anisotropy values were analyzed by nonlinear regression in GraphPad Prism 5. The $K_{d}$ values reported are the average obtained from three independent experiments.

Electrophoretic Mobility Shift Assays-Affinities were estimated by mixing FACT or its domains with recombinant yeast or Xenopus histones and then subjecting the samples to native PAGE, as described $(4,24)$.

\section{RESULTS AND DISCUSSION}

Spt16-M Adopts a Double PH Domain Structure That Resembles Pob3-M and Rtt106-The design of Spt16-M constructs for crystallization trials was guided by the results of limited proteolysis experiments and inspection of the amino acid sequence. In addition to 8 residues of non-native sequence at the $\mathrm{N}$ terminus, the construct that crystallized spans Spt16 residues Gly-675 to Asp-958 and lacks residues Gly-774-Ser-780, which were predicted to lie within a flexible loop. Experimental phases were determined by the SAD method, and the model was refined to $R_{\text {work }} / R_{\text {free }}$ values of $17.7 \% / 19.7 \%$ against $1.9 \AA$ resolution native data. There was one Spt16-M molecule in the asymmetric unit.

Spt16-M adopts a double PH domain structure (Fig. 1), in which the PH1 and PH2 domains both conform to the canoni- 

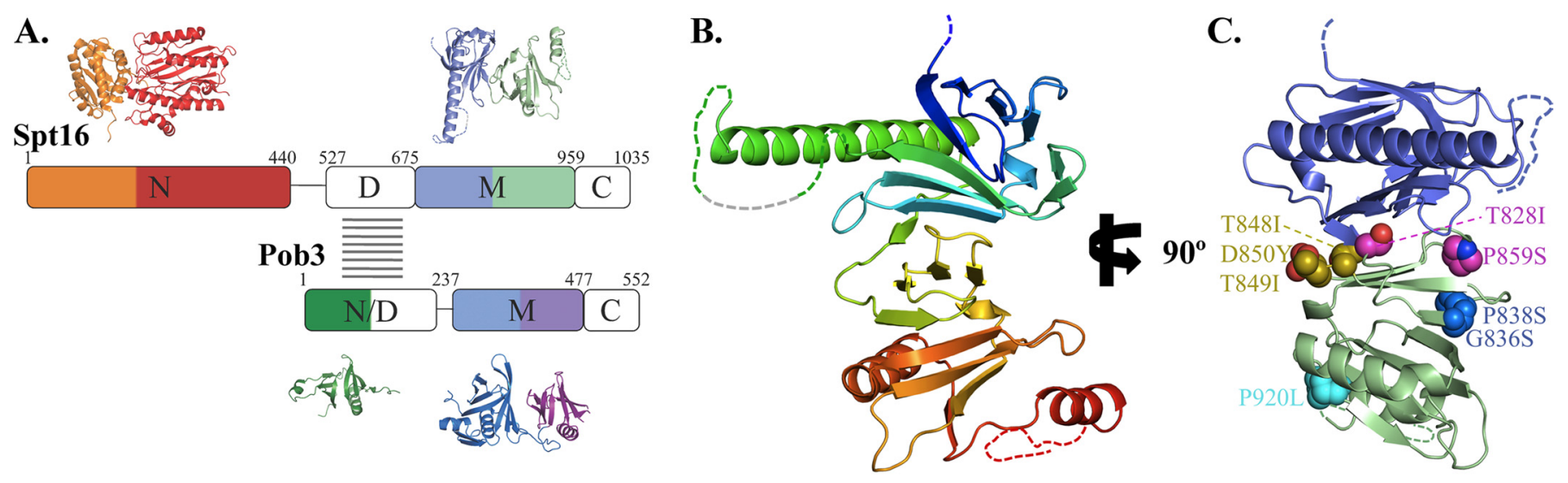

D.

675

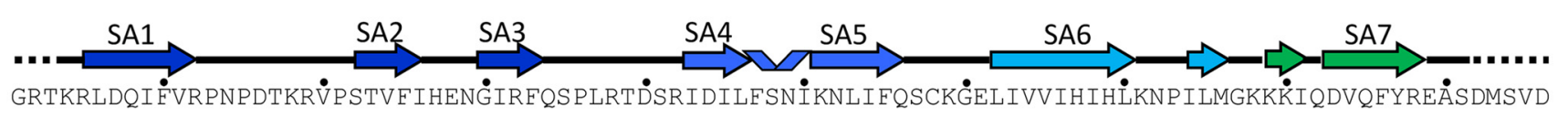

771 ETGGGRRGQS

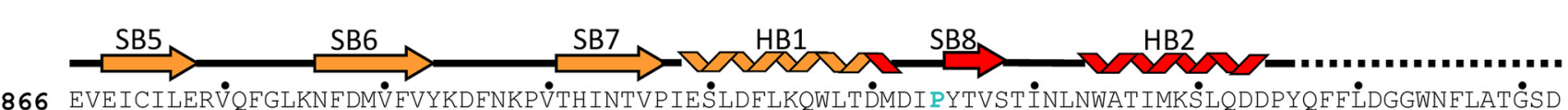

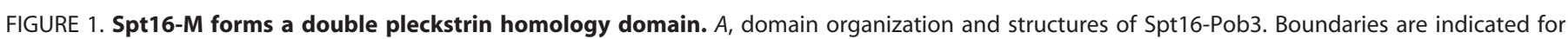

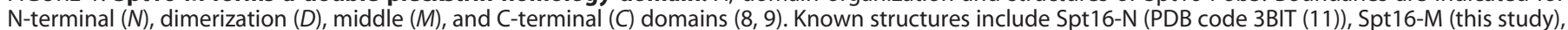

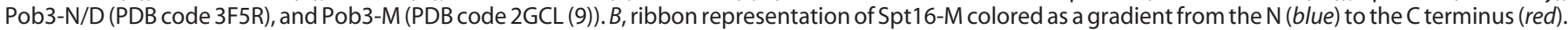

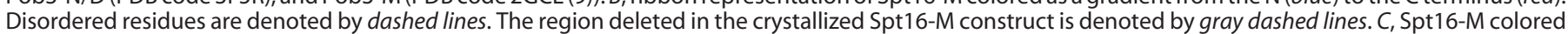

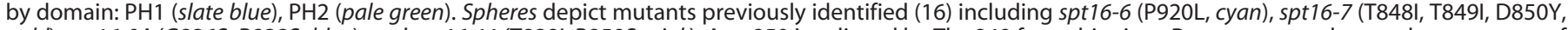

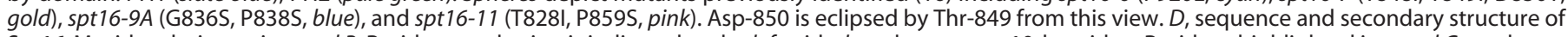

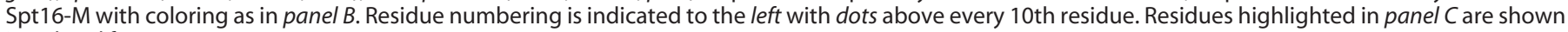
in colored font.

cal PH domain architecture, which comprises a seven-stranded antiparallel $\beta$-barrel that buries a hydrophobic core and has one end capped by a C-terminal $\alpha$-helix (25). Secondary structure elements are named according to the canonical $\mathrm{PH}$ domain scheme $(26,27)$. Although PH domains often form independent structural units, the PH1 and PH2 domains of Spt16-M associate with each other through an extensive interaction interface to form a single, apparently rigid double $\mathrm{PH}$ structural unit, an arrangement also found in Pob3-M and Rtt106 (9, 13, 14, 28). The impression of a rigid association is reinforced by the observation that the PH1-PH2 interface buries several hydrophobic residues, including Ile-728, Val-739, Met-846, Val-853, Leu855, Ile-856, and Phe-860. Moreover, although they share only $\sim 14 \%$ sequence identity, Spt16-M overlaps with Pob3-M (PDB code 2GCL, Ref. 9) and Rtt106-PH (PDB code 3FSS, Ref. 13) with root mean square deviations (r.m.s.d.) of $1.7 \AA$ (over 156 residues) and $2.8 \AA$ (over 143 residues), respectively (Fig. $2 A$ ).

The most striking differences among Spt16-M, Pob3-M, and Rtt106 map to PH1. Pob3-M and Rtt106 each have an additional helix (H2), and loops that extend above PH1 that are not found in Spt16-M or in canonical PH domains (Fig. 2A). Spt16-M also includes features not found in Pob3-M, Rtt106, or canonical PH domains, including an unusually long helix (HA1) in PH1 (3.5 turns longer than the equivalent helix in Pob3 or Rtt106) and an additional helix (HB2) near the $\mathrm{C}$ terminus of $\mathrm{PH} 2$.
Spt16-M Binds Histones H3-H4-To the best of our knowledge, Pob3-M and Rtt106 are the only other double PH domain structures determined, and both are reported to bind to histones H3-H4 $(13,14,28)$. We therefore used an electrophoretic mobility shift assay (EMSA) to determine that Spt16-Pob3 binds to yeast $\mathrm{H} 3-\mathrm{H} 4$ histones with an apparent affinity of $\sim 150$ nм (Fig. 2C) and to Xenopus laevis H3-H4 somewhat less tightly, at $\sim 600 \mathrm{~nm}$ (data not shown). This is consistent with previous studies using a fluorescence shift assay that showed that human FACT binds to Xenopus laevis histones with an affinity of $685 \mathrm{~nm}$ (5). We also examined the binding of Spt16Pob3 to H2A-H2B and, consistent with a report that human FACT binds Xenopus laevis H2A-H2B with an affinity of $31 \mathrm{~nm}$ (5), we determined that yeast Spt16-Pob3 binds yeast H2A-H2B with an affinity of $\sim 15 \mathrm{~nm}$ and binds to Xenopus laevis H2A$\mathrm{H} 2 \mathrm{~B}$ with an affinity of $\sim 50 \mathrm{~nm}$. Intact Spt16-Pob3 therefore has measurable affinity for both $\mathrm{H} 2 \mathrm{~A}-\mathrm{H} 2 \mathrm{~B}$ and $\mathrm{H} 3-\mathrm{H} 4$ histones.

To test whether Spt16-M contributes to the binding of Spt16-Pob3 to histones, we attempted to quantify the interaction with recombinant Spt16-M proteins in the EMSA, but did not observe complex formation with either yeast or X. laevis $\mathrm{H} 3-\mathrm{H} 4$ histones (data not shown). We therefore developed a more sensitive fluorescence anisotropy (FA) assay, which demonstrated that Spt16-M binds X. laevis $\mathrm{H} 3-\mathrm{H} 4$ with an affinity of $\sim 2.5 \mu \mathrm{M}$ (Fig. 2D), at least 4-fold weaker than the Spt16-Pob3 interaction with the same histones determined by EMSA. Yeast 

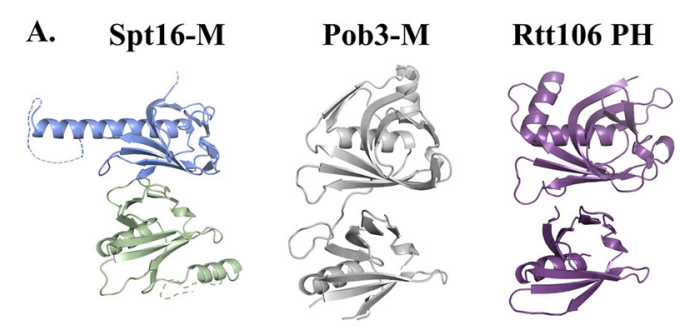

B.
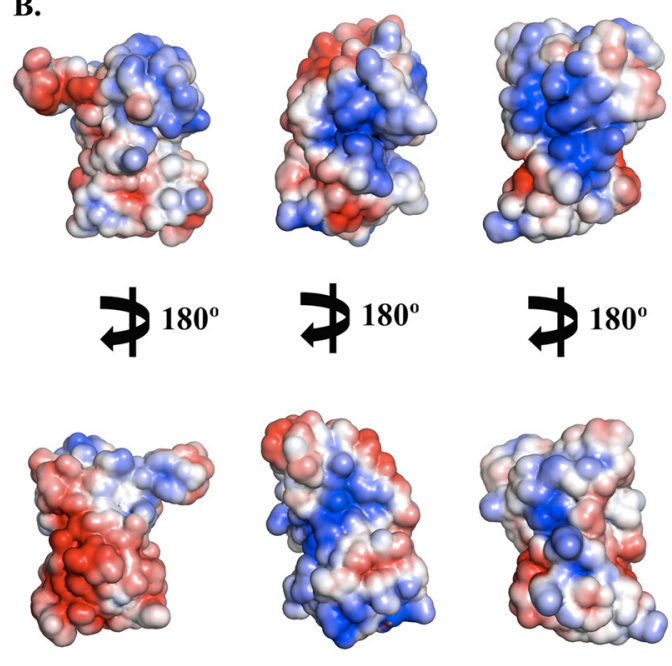

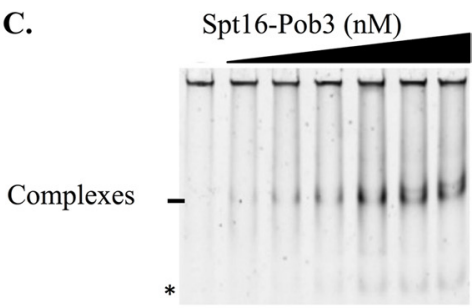

D.
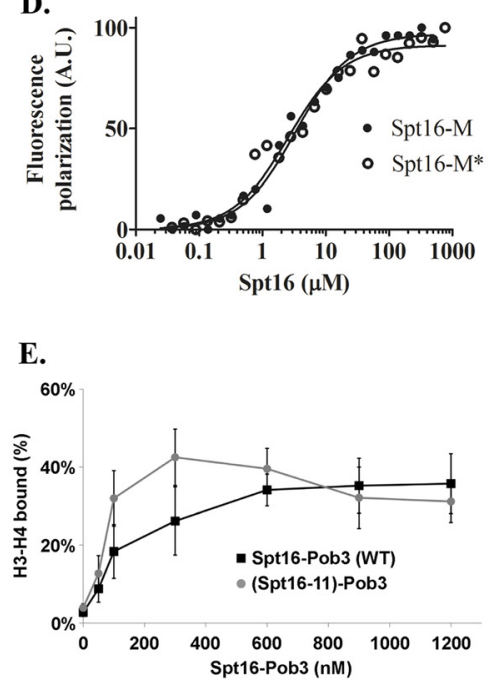

FIGURE 2. Spt16-M is similar to other double PH domains and contributes to binding H3-H4. A, comparison of Spt16-M with the double PH domains of Pob3-M (gray, PDB code 2GCL, Ref. 9) and Rtt106 (purple, PDB code 3FSS, Ref. 13). B, electrostatic surface potential (-3 to 3 kT) of Spt16-M, Rtt106-PH, and Pob3-M. Molecular surface charge maps were prepared using PyMOL APBS tools (10) using a $2 \AA$ solvent radius. C, EMSA showing the mobility of Oregon green-labeled Xenopus laevis H3-H4 in the absence (first lane) and presence of Spt16-Pob3 (50-1200 nM). Complexes of Spt16-Pob3 bound to H3-H4 are indicated. The asterisk indicates a minor complex form of unknown composition with identical dose-response characteristics as the main form. $D$, binding of Spt16-M and Spt16-M* (includes residues Gly-774-Ser-780) to S. cerevisiae H3-H4. Normalized fluorescence polarization values are plotted as a function of increasing concentrations of Spt16. A.U., absorbance units. E, quantitation of an EMSA (performed as in panel C) for $\mathrm{H} 3-\mathrm{H} 4$ binding to Spt16-Pob3 and (Spt16-11)-Pob3. Error bars indicate standard error.

H3-H4 did not yield interpretable results using the FA assay, and we have been unable to achieve adequate concentrations of full-length Spt16-Pob3 to test the interaction with X. laevis H3-H4 by FA, so we cannot compare the affinities directly using the same assay. However, the failure of Spt16-M to form complexes by EMSA and the weaker binding to $\mathrm{H} 3-\mathrm{H} 4$ detected by FA indicate that Spt16-M contributes to H3-H4 binding but is responsible for only part of the binding affinity of Spt16-Pob3 for $\mathrm{H} 3-\mathrm{H} 4$.

We also asked whether Spt16-M contributes to the binding of Spt16-Pob3 to H2A-H2B, but were unable to detect binding with either yeast or X. laevis H2A-H2B by EMSA. No reliable binding signal was observed by FA with yeast $\mathrm{H} 2 \mathrm{~A}-\mathrm{H} 2 \mathrm{~B}$, although signs of a weak interaction with $X$. laevis $\mathrm{H} 2 \mathrm{~A}-\mathrm{H} 2 \mathrm{~B}$ were detected at the highest concentrations of Spt16-M we could achieve, suggesting the potential for binding with an affinity weaker than $50 \mu \mathrm{M}$. Even if this interaction is physiologically relevant, it is much weaker than the $\sim 15-50 \mathrm{~nm}$ interactions detected by EMSA or fluorescence shift with Spt16-Pob3 or human FACT. We conclude that Spt16-M does not contribute significantly to the binding of Spt16-Pob3 to H2A-H2B.

Our results are consistent with reports that the Spt16-N (12) and Pob3-M $(13,14)$ domains also bind histones H3-H4, and they support a model in which the individual domains of Spt16 and Pob3 bind cooperatively with $\mathrm{H} 3-\mathrm{H} 4$ in the context of
FACT. Notably, Rtt106 forms a homodimer through association of two N-terminal domains, which suggests that the two double PH domains in an Rtt106 homodimer might bind the same H3-H4 tetramer cooperatively (13). It is attractive to speculate that this inferred architecture also promotes affinity in the Spt16-Pob3 heterodimer.

Although co-crystal structures of histones bound to a double $\mathrm{PH}$ domain have not yet been reported, important binding determinants on Rtt106 and Pob3 have been identified. These include basic surfaces in Rtt106 and Pob3 that are important for binding dsDNA (28) and histones (14), and a loop in Rtt106 that enables it to recognize acetylated H3K56 (13). Spt16-M lacks the basic patch described in Rtt106 and Pob3-M, but instead has a prominent acidic patch in $\mathrm{PH} 2$ that is less apparent in Pob3-M or Rtt106 (Fig. 2B). Thus, although these related chaperones use similar architectures to bind $\mathrm{H} 3-\mathrm{H} 4$, the detailed mechanisms may differ.

Published genetic data are consistent with the functional importance of the Spt16-M-H3-H4 interaction (Fig. 3, $A-D$ ). The L61W mutation in histone H3 disturbs the structure of nucleosomes, presumably by destabilizing the interface between $\mathrm{H} 3$ and $\mathrm{H} 4$ (29). Growth defects caused by this mutation can be suppressed by mutations in $\operatorname{SPT} 16(15,29)$ that map to Spt16-M. Although the suppression mechanism is not known, these observations indicate a functional link between 
A.

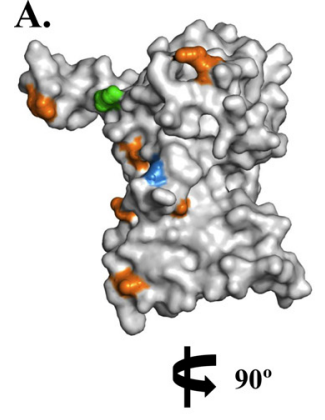

C.

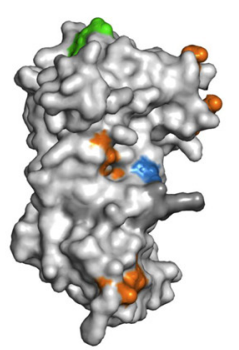

E.

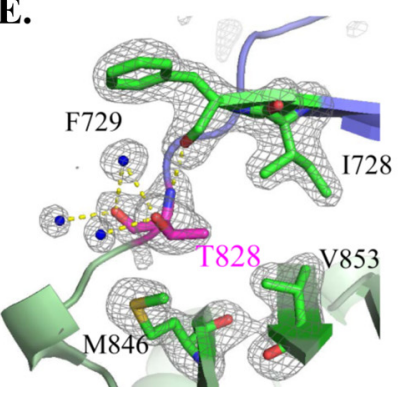

B.

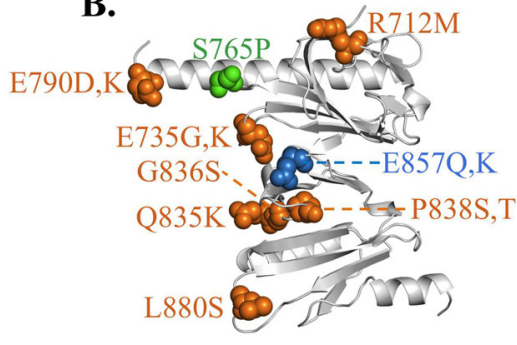

D.

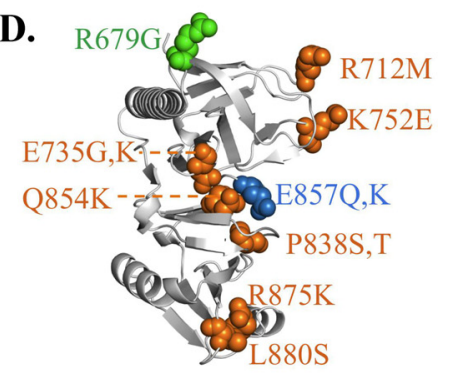

F.

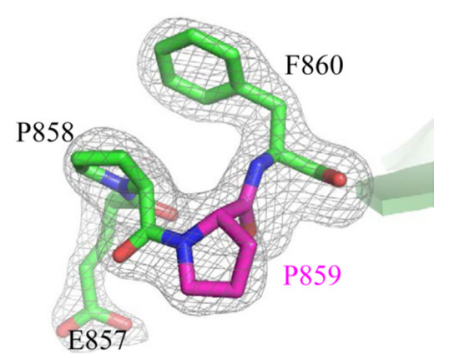

FIGURE 3. Location of previously identified SPT16 mutations and the structural basis of the spt16-11 phenotype. $A-D$, surface ( $A$ and $C$ ) and sphere $(B$ and $D$ ) representations of Spt16-M mutations that suppress H3-L61W (orange (15)) or cause derepression of SER (green (18)). Glu-857 (blue) was identified in both studies, mutated to either a Gln (15), or a Lys (18). $E$ and $F_{,} F_{o}-F_{c}$ omit map contoured at $3 \times$ r.m.s.d. around residues Thr-828 and Pro-859. Protein and solvent in the figure were deleted from the model, and random $0.1 \AA$ shifts were applied to the rest of the structure followed by two cycles of refinement.

Spt16-M and histones H3-H4. Similarly, most of the mutations in SPT16 that were found to cause derepression of SER3, and are therefore implicated in reassembly of nucleosomes after passage of RNA polymerase, also mapped to Spt16-M (18). This further supports an important role for this domain in restraining dispersal of nucleosomal components during transcription, presumably by binding directly to those components.

Structural Basis of the Spt16-11 Phenotype-Four previously identified mutants that display the $\mathrm{Spt}^{-}$phenotype, and in some cases are temperature-sensitive and/or hydroxyurea-sensitive (16), map to Spt16-M (Fig. 1C). Of these, the spt16-11 allele has been used extensively for genetic analysis because strains with this mutation display the full range of defects associated with FACT mutations (30). spt16-11 includes two mutations, T828I and P859S, which are both necessary to produce these phenotypes.

Thr-828 is located in the loop connecting PH1 and PH2. The threonine side chain methyl group projects into a hydrophobic pocket (Fig. 3E), whereas the side chain hydroxyl lies in a depression on the surface of Spt16 that is lined by polar side

chains, where it forms H-bonds with ordered water molecules. Simple modeling suggests that isoleucine, which substitutes for Thr-828 in the Spt16-11 mutant, will destabilize the local protein structure by disrupting both packing of the hydrophobic pocket and hydrogen-bonding interactions near the protein surface.

Substitution of Pro-859 for serine, which is the other mutation that occurs in Spt16-11, is also expected to destabilize the protein structure. This residue is highly conserved from yeasts to human. It is located in the loop connecting SB3 and SB4 and adopts a cis conformation (Fig. $3 F$ ), which indicates that its substitution, including by serine, will destabilize the local protein structure.

Inspection of the structure suggests that the T828I and P859S substitutions are unlikely to be catastrophic for the overall protein conformation, but that they will each cause some structural instability by disrupting the local conformation. Their locations, on opposite sides of the waist of the double $\mathrm{PH}$ fold at the interface between $\mathrm{PH} 1$ and $\mathrm{PH} 2$, suggest at least two possible mechanisms by which they might perturb Spt16 function. One possibility is that in combination they might destabilize the overall structure to the extent that its functions are substantially impaired. A reduction in conformational stability in Spt16-11 is consistent with the observation that the level of Spt16 protein is reduced to about $20 \%$ of wild type after $3 \mathrm{~h}$ at the nonpermissive temperature of $37^{\circ} \mathrm{C}$ and is about $70 \%$ of wild type in cells growing at $24^{\circ} \mathrm{C}(11)$. It is unlikely, however, that all of the spt16-11 phenotypes result from reduced protein levels because similar levels are observed for an Spt16-G132D mutant, which does not cause a phenotype under conditions where spt16-11 strain display hydroxyurea sensitivity $(11,16)$. Thus, an attractive second possibility is that the Spt16-11 mutations perturb a specific function such as a binding interaction. Consistent with this possibility, purified FACT with the Spt16-11 (T828I, P859S) mutations is functional, but less effective than WT FACT at promoting restriction endonuclease accessibility of nucleosomal DNA (17). This defect was corrected by an H2B-A84D mutation that weakens the interface between $\mathrm{H} 2 \mathrm{~B}$ and $\mathrm{H} 3-\mathrm{H} 4$ in nucleosomes. This suggests that Spt16-11 protein does not efficiently induce or trap reorganized nucleosomes, and this defect can be compensated by a second mutation that makes nucleosomes more prone to being reorganized (17). Together, the data suggest that proper juxtaposition of PH1 and PH2 of Spt16-M is important for maintaining both the stability and the appropriate functioning of Spt16.

Similarly to Spt16-11, inspection of the structure suggests that all of the mutations illustrated in Fig. $1 C$ will be slightly destabilizing but are unlikely to have a catastrophic effect on the overall protein conformation. The most innocuous-appearing of these mutations is P920L, which may destabilize the structure by placing a large hydrophobic side chain in a solventexposed environment, modestly disrupting interactions with other residues at the protein surface, and perhaps by destabilizing a kink in the protein chain that is stabilized by a proline. For this mutant in particular, it is tempting to speculate that the phenotype results from disruption of a functional interaction because it is close to a loop that has been implicated in $\mathrm{H} 3-\mathrm{H} 4$ 
binding by Pob3 $(13,14)$ and near residues that display a change in chemical shift upon interaction with H3-H4 by Rtt106 (13).

Spt16-11 Does Not Disrupt Histone H3-H4 Binding-An obvious hypothesis to explain the defects observed in spt16-11 mutants was that this mutation disturbs the interaction between FACT and H3-H4. We tested this idea using the EMSA to quantify binding of (Spt16-11)-Pob3 to H3-H4. However, the results showed instead that the mutant version of FACT retains affinity for histones $\mathrm{H} 3-\mathrm{H} 4$ and even seems to increase it slightly (Fig. 2E). We do not favor the possibility that the increased affinity, which is small but reproducible, is the primary defect caused by the spt16-11 mutation because this gain of function should lead to genetic dominance of this allele, whereas all phenotypes it causes have been found to be fully recessive. We also note that, consistent with the previous conclusion that Spt16-M does not contribute substantially to the interaction between Spt16-Pob3 and H2A-H2B, (Spt16-11)Pob3 bound to H2A-H2B with the same $\sim 15$ nM affinity observed with WT Spt16-Pob3. The physiological defects caused by spt16-11 therefore do not appear to involve loss of binding affinity between Spt16-Pob3 and H3-H4, but may instead be related to a defect in inducing or stabilizing the reorganized form of nucleosomes, as suggested previously (17).

Implications for Future Studies-Our finding that Spt16-M adopts a double $\mathrm{PH}$ domain structure and binds histones H3-H4 reveals a striking similarity to Pob3-M and Rtt106. Despite the overall similarity, differences among the three structures, including the additional secondary structural elements and distribution of surface electrostatic potential, confound efforts to propose a common mechanism of binding. The extent to which common binding modes are deployed, and the extent to which the recognition of lysine acetylation that has been characterized for Rtt106 is mirrored or missing in Pob3 and Spt16, are important questions for future studies. In addition to understanding how the individual double $\mathrm{PH}$ domains bind histones $\mathrm{H} 3-\mathrm{H} 4$, it will be important to understand how the two halves of the homodimeric structure of Rtt106 and the heterodimeric structure of Spt16-Pob3 collaborate to bind histones H3-H4. Relevant questions include: do the dimers make bidentate interactions with the same $(\mathrm{H} 3-\mathrm{H} 4)_{2}$ tetramer while it is in a canonical nucleosome structure, do they promote disassembly of the tetramer from other nucleosomal components, or do they promote disassembly of the $(\mathrm{H} 3-\mathrm{H} 4)_{2}$ tetramer into dimers? The locations of residues identified as important in various genetic screens (15, $16,18,29)$ are consistent with the conclusion that Spt16-M surfaces participate in extensive interactions, including those relevant for the functional interaction with $\mathrm{H} 3-\mathrm{H} 4$. Beyond the H3-H4 interactions, it will also be important to understand how Spt16-Pob3 interacts with other nucleosomal components. In particular, Spt16-Pob3 binds histones H2A-H2B (see above and Refs. 5 and 6), but we found that this activity does not reside substantially within the Spt16-M domain. Understanding how interactions with $\mathrm{H} 2 \mathrm{~A}-\mathrm{H} 2 \mathrm{~B}$ and $\mathrm{H} 3-\mathrm{H} 4$ are coordinated will likely be central to understanding the biological mechanism of Spt16-Pob3/FACT.

\section{REFERENCES}

1. Formosa, T. (2012) The role of FACT in making and breaking nucleosomes. Biochim. Biophys. Acta 1819, 247-255

2. Winkler, D. D., and Luger, K. (2011) The histone chaperone FACT: structural insights and mechanisms for nucleosome reorganization. J. Biol. Chem. 286, 18369-18374

3. Belotserkovskaya, R., Saunders, A., Lis, J. T., and Reinberg, D. (2004) Transcription through chromatin: understanding a complex FACT. Biochim. Biophys. Acta 1677, 87-99

4. Xin, H., Takahata, S., Blanksma, M., McCullough, L., Stillman, D. J., and Formosa, T. (2009) yFACT induces global accessibility of nucleosomal DNA without H2A-H2B displacement. Mol. Cell 35, 365-376

5. Winkler, D. D., Muthurajan, U. M., Hieb, A. R., and Luger, K. (2011) Histone chaperone FACT coordinates nucleosome interaction through multiple synergistic binding events. J. Biol. Chem. 286, 41883-41892

6. Orphanides, G., Wu, W. H., Lane, W. S., Hampsey, M., and Reinberg, D. (1999) The chromatin-specific transcription elongation factor FACT comprises the human SPT16/CDC68 and SSRP1 proteins. Nature 400, $284-288$

7. Jamai, A., Puglisi, A., and Strubin, M. (2009) Histone chaperone Spt16 promotes redeposition of the original h3-h4 histones evicted by elongating RNA polymerase. Mol. Cell 35, 377-383

8. O’Donnell, A. F., Brewster, N. K., Kurniawan, J., Minard, L. V., Johnston, G. C., and Singer, R. A. (2004) Domain organization of the yeast histone chaperone FACT: the conserved N-terminal domain of FACT subunit Spt16 mediates recovery from replication stress. Nucleic Acids Res. 32, 5894-5906

9. VanDemark, A. P., Blanksma, M., Ferris, E., Heroux, A., Hill, C. P., and Formosa, T. (2006) The structure of the yFACT Pob3-M domain, its interaction with the DNA replication factor RPA, and a potential role in nucleosome deposition. Mol. Cell 22, 363-374

10. Baker, N. A., Sept, D., Joseph, S., Holst, M. J., and McCammon, J. A. (2001) Electrostatics of nanosystems: application to microtubules and the ribosome. Proc. Natl. Acad. Sci. U.S.A. 98, 10037-10041

11. VanDemark, A. P., Xin, H., McCullough, L., Rawlins, R., Bentley, S., Heroux, A., Stillman, D. J., Hill, C. P., and Formosa, T. (2008) Structural and functional analysis of the Spt16p N-terminal domain reveals overlapping roles of yFACT subunits. J. Biol. Chem. 283, 5058-5068

12. Stuwe, T., Hothorn, M., Lejeune, E., Rybin, V., Bortfeld, M., Scheffzek, K., and Ladurner, A. G. (2008) The FACT Spt16 "peptidase" domain is a histone H3-H4 binding module. Proc. Natl. Acad. Sci. U.S.A. 105, $8884-8889$

13. Su, D., Hu, Q., Li, Q., Thompson, J. R., Cui, G., Fazly, A., Davies, B. A., Botuyan, M. V., Zhang, Z., and Mer, G. (2012) Structural basis for recognition of H3K56-acetylated histone H3-H4 by the chaperone Rtt106. Nature 483, 104-107

14. Zunder, R. M., Antczak, A. J., Berger, J. M., and Rine, J. (2012) Two surfaces on the histone chaperone Rtt106 mediate histone binding, replication, and silencing. Proc. Natl. Acad. Sci. U.S.A. 109, E144-E153

15. Myers, C. N., Berner, G. B., Holthoff, J. H., Martinez-Fonts, K., Harper, J. A., Alford, S., Taylor, M. N., and Duina, A. A. (2011) Mutant versions of the S. cerevisiae transcription elongation factor Spt16 define regions of Spt16 that functionally interact with histone H3. PLoS One 6, e20847

16. Formosa, T., Eriksson, P., Wittmeyer, J., Ginn, J., Yu, Y., and Stillman, D. J. (2001) Spt16-Pob3 and the HMG protein Nhp6 combine to form the nucleosome-binding factor SPN. EMBO J. 20, 3506-3517

17. McCullough, L., Rawlins, R., Olsen, A., Xin, H., Stillman, D. J., and Formosa, T. (2011) Insight into the mechanism of nucleosome reorganization from histone mutants that suppress defects in the FACT histone chaperone. Genetics 188, 835-846

18. Hainer, S. J., Charsar, B. A., Cohen, S. B., and Martens, J. A. (2012) Identification of mutant versions of the Spt16 histone chaperone that are defective for transcription-coupled nucleosome occupancy in Saccharomyces cerevisiae. G3 (Bethesda) 2, 555-567

19. Studier, F. W. (2005) Protein production by auto-induction in high density shaking cultures. Protein Expr. Purif. 41, 207-234

20. Otwinowski, Z., and Minor, W. (1997) Processing of x-ray diffraction data 
collected in oscillation mode. Methods Enzymol. 276, 307-326

21. Adams, P. D., Afonine, P. V., Bunkóczi, G., Chen, V. B., Davis, I. W., Echols, N., Headd, J. J., Hung, L. W., Kapral, G. J., Grosse-Kunstleve, R. W., McCoy, A. J., Moriarty, N. W., Oeffner, R., Read, R. J., Richardson, D. C., Richardson, J. S., Terwilliger, T. C., and Zwart, P. H. (2010) PHENIX: a comprehensive Python-based system for macromolecular structure solution. Acta Crystallogr. D Biol. Crystallogr. 66, 213-221

22. Emsley, P., Lohkamp, B., Scott, W. G., and Cowtan, K. (2010) Features and development of Coot. Acta Crystallogr. D Biol. Crystallogr. 66, 486-501

23. DeLano, W. L. (2012) The PyMOL Molecular Graphics System, version 1.5.0.4, Schrödinger, LLC, New York

24. Rhoades, A. R., Ruone, S., and Formosa, T. (2004) Structural features of nucleosomes reorganized by yeast FACT and its HMG box component, Nhp6. Mol. Cell. Biol. 24, 3907-3917

25. Lemmon, M. A. (2004) Pleckstrin homology domains: not just for phosphoinositides. Biochem. Soc. Trans. 32, 707-711
26. Yoon, H. S., Hajduk, P. J., Petros, A. M., Olejniczak, E. T., Meadows, R. P., and Fesik, S. W. (1994) Solution structure of a pleckstrin-homology domain. Nature 369, 672-675

27. Ferguson, K. M., Lemmon, M. A., Schlessinger, J., and Sigler, P. B. (1994) Crystal structure at 2.2 Ä resolution of the pleckstrin homology domain from human dynamin. Cell 79, 199-209

28. Liu, Y., Huang, H., Zhou, B. O., Wang, S. S., Hu, Y., Li, X., Liu, J., Zang, J., Niu, L., Wu, J., Zhou, J. Q., Teng, M., and Shi, Y. (2010) Structural analysis of Rtt106p reveals a DNA binding role required for heterochromatin silencing. J. Biol. Chem. 285, 4251-4262

29. Duina, A. A., and Winston, F. (2004) Analysis of a mutant histone $\mathrm{H} 3$ that perturbs the association of Swi/Snf with chromatin. Mol. Cell. Biol. 24, $561-572$

30. Clark-Adams, C. D., Norris, D., Osley, M. A., Fassler, J. S., and Winston, F. (1988) Changes in histone gene dosage alter transcription in yeast. Genes Dev. 2, 150-159 